\title{
Externalities in Charitable Crowdfunding
}

\author{
Deserina Sulaeman \\ Singapore Management University \\ deserinas.2015@phdis.smu.edu.sg
}

\begin{abstract}
This study examines how the existence of multiple charitable campaigns supporting similar causes on the same platform influence the donations received by each campaign. The existence of multiple campaigns supporting similar causes can have negative externalities as they compete for donors. However, the existence of additional campaigns can also result in positive externalities as potential donors benefit from a larger variety of projects to choose from. This study observes negative and positive externalities from the existence of multiple campaigns supporting similar causes. An increase in the number of campaigns leads to a decrease in the average donation received by each campaign, indicating that the additional campaigns do not necessarily expand the market. However, when some of the campaigns perform exceptionally well, other campaigns - including non-top performing campaigns - receive more donation, highlighting the market expansion effect of the presence of successful campaigns on a platform.
\end{abstract}

\section{Introduction}

Crowdfunding has transformed charitable fundraising practices [8]. Charitable fundraisers, even inexperienced ones, can now start new charitable campaigns on crowdfunding platforms and reach a large set of potential donors around the world at low fundraising costs. Therefore, it is not surprising that more and more charitable fundraisers turn to crowdfunding platforms as a viable fundraising channel in the recent years. Indeed, GoFundMe, a charity-focused crowdfunding platform and the focus of this study, is the fourth-ranked crowdfunding platform based on site traffics as recorded by Alexa.com [12]. It trails only Kickstarter, Indiegogo,

\footnotetext{
${ }^{1}$ Some campaigns on GoFundMe support causes that are not typically associated with charities, such as funding memorial funds for an individual, funding a personal trip, funding a wedding, etc.
}

and Patreon, which are not charity-focused. Fundraisers on GoFundMe have raised more than US\$ 3 billion since the platform's inception in 2010 [1].

With the increasing popularity of charitable fundraising on crowdfunding platforms, many charitable crowdfunding campaigns are likely to share similar causes - that is, supporting the relief efforts associated with a particular natural disaster event - on the same platform. Indeed, the dataset utilized in this study includes at least 850 campaigns posted on GoFundMe in support of the relief efforts of victims of Hurricane Matthew that made its landfall in South Carolina, USA on October 8, 2016. Similarly, over 2,600 campaigns were posted on GoFundMe in response to Hurricane Harvey that made its landfall in the Texas Gulf Coast on August 25, 2017. This study examines how the existence of multiple charitable campaigns supporting similar causes on the same crowdfunding platform influence the donations received by these campaigns.

This study examines campaigns aiming to support relief efforts of natural disaster events. This focus has two benefits. First, the analyses mitigate the (potentially large) variation in the worthiness of causes supported by various campaigns on charitable crowdfunding platforms. ${ }^{1}$ The campaigns examined in this study share the same focus of natural disaster relief efforts, and therefore their causes are quite similar. Second, this focus allows for comparisons with findings from studies on competition in markets where corporations or institutions offer similar products (i.e., products that are close substitutes) and compete against each other for buyers.

To the best of my knowledge, this is the first study to examine how the presence of other charitable campaigns influences the success of campaigns on crowdfunding platforms. Specifically, this study investigate whether the presence of other campaigns supporting similar causes result in both positive and negative externalities.

An example of such campaigns is

https://www.gofundme.com/AmieeVacationFunds. 
Existing studies on other markets have documented that the presence of additional firms have negative externalities (e.g., [11]). These negative externalities stem from business stealing among firms in that market. In the charitable crowdfunding context, faced with budget constraints, donors are unlikely to contribute to all campaigns with similar causes. As a result, these campaigns compete against each other for potential donors' money.

However, existing studies have also identified a potential channel of positive externalities (e.g., [2], [3], [7], [10], [15], and [16]). Crowdfunding platforms are two-sided markets, in which agents from one side of the market benefit from increased interactions with agents from the other side of the market, creating positive cross-group externality ([3] and [15]) that potentially increases the size of the market.

In the charitable crowdfunding context, more donors may be attracted to a particular platform when the number of campaigns that donors can choose from in that platform increases. The additional campaigns offer a larger variety of projects that potential donors can choose from. However, the larger variety does not necessarily mean that donors would have an easier time identifying campaigns that maximize their utilities. In order to increase their chances of finding such campaigns, donors would be looking for signals that a platform hosts high-quality campaigns. When top campaigns on a platform perform particularly well, potential donors are likely to use this as a quality signal, not only of those top campaigns, but also of the overall quality of campaigns hosted on that platform [16].

The empirical analyses in this study include two sets of control variables to mitigate potential endogeneity concerns. First, to capture time-invariant heterogeneity across campaigns, campaign fixed effects are included in all analyses at the campaign level. Second, a vector of control variables are included in the empirical model. To the extent that this vector includes relevant factors that may affect both campaign outcomes and the presence of additional campaigns, the inclusion of these control variables helps to mitigate potential omitted variable concerns.

This study observes both negative and positive externalities from the presence of other charitable campaigns supporting similar causes on the donations received by each campaign. This study finds that an increase in the number of campaigns supporting relief efforts for a particular natural disaster event leads to less donation received by those campaigns. This is consistent with the business stealing effects of additional campaigns. This observed negative externality suggests that market expansion does not occur merely because more campaigns supporting similar causes are available on the platform.

However, when the top performing campaigns supporting relief efforts for a particular natural disaster event perform exceptionally well in obtaining donations or social media mentions (relative to other campaigns sharing the same event), other campaigns supporting relief efforts for the same event receive more donations. This finding is consistent with the quality-based market expansion hypothesis presented in [10] and [16]. This positive externality is experienced by a wide range of campaigns, including non-top performing campaigns. This indicates that market expansion occurs when the top campaigns perform exceptionally well relative to other campaigns on the platform.

A further analysis of the market shares of topperforming versus non-top performing campaigns indicates that neither the positive nor negative externalities has a significant effect on the distribution of donations among the top performing campaigns. However, for the non-top performing campaigns, while they receive more donations when the top performing campaigns perform exceptionally well, they still lose some of their market shares.

\section{Hypotheses development}

Existing studies have documented both negative and positive externalities from new entrants (e.g., [2], [3], [7], [10], [11], [15], and [16]). A study of peer-topeer microloans by [11] documented a negative effect of the presence of other campaigns, particularly among close substitutes, on funding speed. Their findings suggest that similar projects on a peer-to-peer microloan platform are stealing lenders from one another.

Using charitable donors' utility function posited by [14], we assume that donors on charitable crowdfunding platforms receive positive utility from two sources: (1) from the joy of making a donation (i.e., warm glow or joy-of-giving) and (2) from the improvement of the well-being of the beneficiaries as the results of the benefits delivered by the fundraisers (i.e., altruism). However, each donor's ability to maximize her/his utility is limited by her/his budget constraint. Given these constraints, donors are unlikely to contribute to all campaigns providing them with positive utility. As a result, campaigns will have to compete with one another to get their donations. Therefore, we expect: 
Hypothesis 1 (Negative Externalities): $A n$ increase in the number of campaigns supporting similar causes on the same platform leads to less donation received by each of those campaigns.

In the context of two-sided markets, such as crowdfunding platforms, agents from one side of the market benefit from their interactions with agents from the other side of the market and vice versa. This positive cross-group externality, as posited by [3] and [15], implies that as the number of agents from one side of the market increases, the number of agents from the other side is likely to increase. As such, the market can expand as a result of new entrants into the market. Assuming that the size of the donors' market is endogenous, [2] posited that the entries of additional non-governmental organizations (NGO) can "wake" additional potential donors through increased collective fundraising efforts. Consistent with this hypothesis, [7] found that entry by high quality movie theaters attracts new patrons to the theaters.

[10] and [16] posit that the quality of the agents from one side of the market also matters in attracting more agents from the other side of the market to the platform. When potential buyers observe the presence of some high quality sellers on a particular platform, they revise their beliefs regarding the quality of all sellers on that particular platform upwards. [16] argues that low quality sellers will not enter a platform dominated by high quality sellers because of the potential cost faced by low quality sellers if their true quality is later revealed to the buyers. As such, potential buyers prefer to transact in a platform dominated by high quality sellers as it increases their likelihood of finding campaigns that maximize their utilities [10].

In this context, the presence of more campaigns may expand the potential donors market, which leads to higher donations received by each campaign on the platform. Consistent with [10] and [16], such market expansion is likely to occur in the presence of high quality campaigns. In most cases, the quality of a campaign is not fully revealed to potential donors. In these cases, donors may look at campaigns' performance in raising funds as a signal of quality [4]. When campaigns on a platform - particularly top campaigns, which are the most visible to potential donors - are doing exceptionally well in raising funds they send a signal of a good quality. This signal spills over to the platform and by extension to other campaigns on the platform, which then attracts more potential donors.

As such, we can expect that when the top campaigns on a platform are performing exceptionally well donors market on the platform is expanded. In this study, the performance of the top campaigns is measured using the portion of the donations received by the top $25 \%$ of highest earning campaigns over the donations received by all campaigns supporting the relief efforts to help victims of a particular natural disaster event of top earning campaigns on a particular platform.

Hypothesis 2 (Positive Externalities): When the top earning campaigns supporting relief efforts of a particular event receive more donations relative to the rest of the campaigns associated with the same event, campaigns associated with that same event receive more donations.

\section{Data description}

This study utilizes a panel dataset containing charitable campaigns on GoFundMe that help victims of natural disaster events. Python codes were utilized to collect publicly available data daily from GoFundMe's website, www.gofundme.com, in 2016 and 2017. The panel dataset contains daily data for at least 50 days after the occurrence of each event of 7,899 unique charitable campaigns helping victims of five major natural disaster events: Hurricane Matthew (September 2016), Hurricane Harvey (August 2017), Hurricane Irma (September 2017), Hurricane Maria (September 2017), and Mexico City earthquake (September 2017).

When queried for a specific natural disaster event, GoFundMe displays at most 500 campaigns related to that event. These displayed campaigns seem to be the most active (i.e., still receiving donations) among campaigns supporting relief efforts for the corresponding natural disaster event. For our analyses, we focus on campaigns that appear on the list displayed by GoFundMe on the previous day $t-1$. This approach allows us to measure the donations received on day $t$ as the difference between the total donation amount in day $t$ and the total donation amount in day $t-1$. Therefore, the analysis in this study employs at most 500 campaigns during each day following a particular natural disaster.

However, we use all campaigns supporting similar causes that have appeared on GoFundMe's list thus far to calculate the independent variables of interest: number of campaigns and the ratios of high- vs. lowperforming campaigns. We do this as donors can still access each active campaign, including those not appearing on GoFundMe's list, using a direct link to the campaign's page. Moreover, donors who have donated to unlisted campaigns can continue to be 
advocates for those campaigns as well as the GoFundMe platform.

The charitable crowdfunding campaigns in the dataset raised a total of US\$ 35.9 million from 327,004 donors by the end of the data collection periods (see Table 1). The dataset includes at least 50 days after the occurrence of each event. The data on Hurricane Matthew, Hurricane Maria, and Mexico City earthquake cover 50 days since the occurrence of each event. The data on Hurricane Harvey cover 57 days after the hurricane made its landfall in Texas. The data on Hurricane Irma cover 59 days since its landfall in Florida. Campaigns on GoFundMe can stay open for a long time even after their funding goals are reached. However, the 50-days observation window seems sufficient to capture the campaigns' life cycle. As shown on Figure 1, over $87 \%$ of the total amounts of donations were received within the first 30 days of the occurrence of the events in our dataset. These campaigns do not receive much donations anymore beyond 30 days after the occurrence of the events they are associated with. Therefore, the 50-day window includes the active period (i.e., first 30 days after the occurrence of each event) as well as the non-active period (i.e., beyond day 30) of fundraising for victims of natural disaster events.

Similarly, Figure 2 shows that most campaigns entered the market within the first 10 days of the occurrence of the events they are associated with. Over $97 \%$ of campaigns in our dataset entered the market within the first 30 days of the occurrence of the events in the dataset. This implies that within the same event, most campaigns are of similar age. The relatively short active period of fundraising is consistent with the urgent nature of natural disaster relief efforts and the short attention span given to such events.

Table 1. Campaign Statistics, by event

\begin{tabular}{l|c|c|c|c}
\hline \multicolumn{1}{c|}{ Event } & Date & $\begin{array}{c}\text { Number of } \\
\text { Campaigns }\end{array}$ & $\begin{array}{c}\text { Total } \\
\text { Donations }\end{array}$ & $\begin{array}{c}\text { Total } \\
\text { Donors }\end{array}$ \\
\hline $\begin{array}{l}\text { Hurricane } \\
\text { Matthew }\end{array}$ & $10 / 16$ & 850 & $\$ 3,007,368$ & 31,214 \\
$\begin{array}{l}\text { Hurricane } \\
\text { Harvey }\end{array}$ & $08 / 17$ & 2,627 & $\$ 19,220,782$ & 169,954 \\
$\begin{array}{l}\text { Hurricane } \\
\text { Irma }\end{array}$ & $09 / 17$ & 2,516 & $\$ 7,110,700$ & 61,925 \\
$\begin{array}{l}\text { Hurricane } \\
\text { Maria }\end{array}$ & $09 / 17$ & 1,906 & $\$ 6,624,402$ & 63,911 \\
$\begin{array}{l}\text { Mexico City } \\
\text { earthquake }\end{array}$ & $09 / 17$ & 133 & $\$ 274,669$ & 2,905 \\
\hline \multicolumn{2}{l}{ Total } & 7,899 & $\$ 35,963,252$ & 327,004 \\
\hline
\end{tabular}

Additionally, the Google Trends index for each of the natural disaster events in the dataset is used to capture the differences in the popularity of those events. Google Trends index is calculated using query share, which is the search volume for a particular query term divided by the total search volume on Google at a given point in time [5]. The Google Trends index of a particular natural disaster event can be utilized as a proxy for how popular that event is because the index represents the relative volume of search for that particular event.

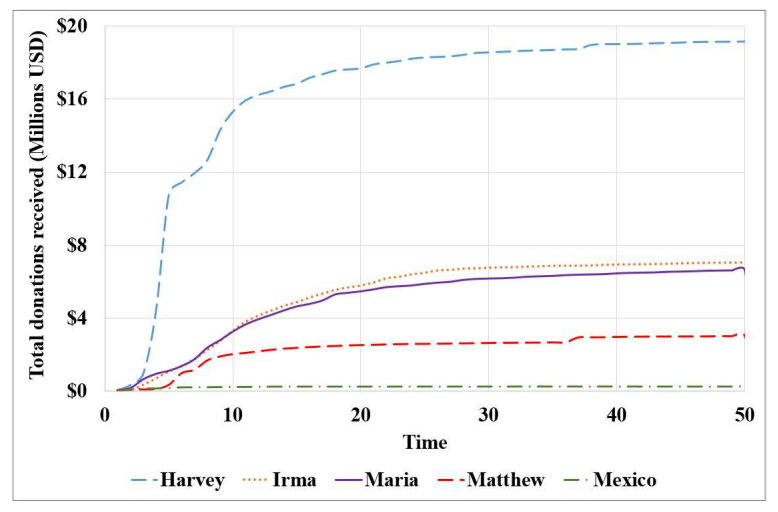

Figure 1. Total donations received, by event and time

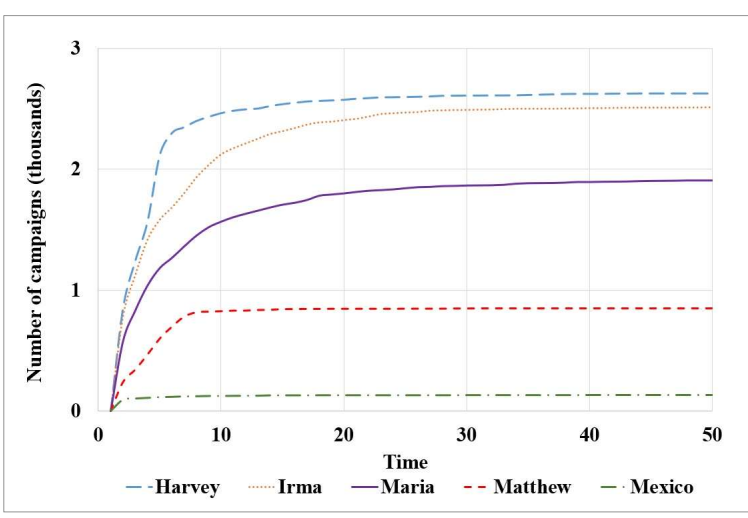

Figure 2. Number of campaigns, by event and time

\section{Empirical model}

The presence of other charitable campaigns supporting similar causes can have both negative and positive externalities for all campaigns with similar causes. The empirical model in Equation 1 is used to test for the existence of negative and positive externalities as specified in Hypotheses 1 and 2. 


$$
\begin{gathered}
\ln \left(\text { DonationRcv }_{t, i}\right)=\alpha_{1} * \text { NumCampaigns } \\
+\alpha_{2} * \text { EarnRatio }_{t-1}+\mathrm{B} * X+\Theta * Z+\varepsilon
\end{gathered}
$$

The dependent variable in the empirical model in Equation 1 (DonationRcv $v_{t, i}$ ) is the amount of donations (in US Dollars) received by campaign $i$ at time $t$. The first independent variable of interest is the number of campaigns supporting the relief efforts of the same natural disaster as campaign $i$ (NumCampaigns N $_{t}$ ) at time $t$. The second independent variable of interest is EarnRatio $_{t-1}$, the ratio of the total donations received by the top $25 \%$ highest earning campaigns supporting the relief efforts of a specific natural disaster event on a particular day over the total donations received by all campaigns focusing on that event on the same day. While the top $25 \%$ campaigns are identified daily, the grouping seems to be quite stable across consecutive days.

Two sets of control variables are included to mitigate potential endogeneity concerns. First, to capture time-invariant heterogeneity across campaigns, campaign fixed effects $(Z)$ are included in the model. As the success of each campaign can depend on its own characteristics as well as the characteristics of the disaster event it is associated with, it is crucial to include campaign fixed effects.

Second, a vector of control variables are included in the empirical model $(X)$ to mitigate potential omitted variable concerns. These control variables include (1) the Google Trends index of the natural disaster event associated with campaign $i$ (GTrends) at time $t-1,(2)$ donations received in the previous time period (DonationRcv at $t-1$ ), and (3) a binary indicator for the first five days since the occurrence of another natural disaster event (NewEvent).

The Google Trends index of the natural disaster event associated with campaign $i$ (GTrends) is included to capture the popularity of the event which may affect the number of potential donors willing to help and the amount of money these donors willing to give to help victims of that particular natural disaster event. It is important to capture the relative popularity of the event in the model to avoid potential endogeneity issue caused by omitted variable biased. More specifically, it is possible that the number of campaigns supporting similar causes and the relative performance of the top campaigns are both driven by the popularity of the particular event associated with these campaigns. The Google Trend index of a natural disaster event is suitable to capture the relative attention received by the event, potentially reflecting the volume of news coverage on that event. Hence, the inclusion of the Google Trend index in the model also alleviate the concern regarding the potential effects of news coverage on the number of campaigns supporting the relief efforts for a particular event and the performance of those campaigns.

The donations received in the previous day is included in the model to control for campaign $i$ 's own momentum from the previous day. The NewEvent binary indicator variable is included in the model to examine the effect of a new natural disaster event during the 50-days fundraising period captured in the dataset. In particular, the NewEvent variable captures the potential effect of the arrival of a new disaster event that attract public interests and can detract potential donors' attention from the focal event. Table 2 displays the summary statistics of the variables included in the model.

The empirical model in Equation 1 is estimated using a panel regression method with campaign fixed effects. Event and time period clustering is also used in the regression to control for within-time-period and within-event correlation.

Table 2. Variables Summary Statistics

\begin{tabular}{lcc}
\hline Variable & Mean & Std. Dev. \\
\hline DonationRcv $_{t}$ & $\$ 169$ & $\$ 1,793$ \\
EarnRatio $_{t-1}$ & 0.88 & 0.10 \\
NumCampaigns $_{t}$ & 1890 & 750 \\
Gtrends $_{t-1}$ & 6.79 & 17.89 \\
\hline
\end{tabular}

\section{Main results}

First, the model in Equation 1 is estimated at an aggregate level. More specifically, we aggregated our dataset based on five natural disaster events across at least 50 time periods for each event. Table 3 shows the regression estimates using the aggregated dataset.

The positive estimate for EarnRatio indicates that the relative success of the top $25 \%$ of campaigns based on donations received has positive externalities on the total donations received by all campaigns supporting relief efforts for a particular natural disaster event in the next day. This result suggests that the success of the top campaigns supporting relief efforts of a particular event is likely to bring in more potential donors to that cause on a platform. Donors who contributed to those top campaigns can reach out to other potential donors and become advocates (i.e., providing additional publicity) for the cause and the platform. 
Table 3. The effects of other campaigns, aggregated by event

\begin{tabular}{lc}
\hline & $\log ($ DonationsRcv $)$ \\
\hline EarnRatio $(\mathrm{t}-1)$ & $17.309 * * *$ \\
& $(5.0971)$ \\
NumCampaigns $(\mathrm{t})$ & 0.002 \\
& $(0.0020)$ \\
\hline Observations & 240 \\
R-squared & 0.532 \\
\hline Note: $* * * \mathrm{p}<0.01, * * \mathrm{p}<0.05, * \mathrm{p}<0.1$. Robust standard errors \\
in parentheses. Coefficients for event fixed effects are \\
suppressed. Control variables are included in the regression, \\
but their estimates are suppressed.
\end{tabular}

Table 4 shows the regression estimates using the non-aggregated dataset (i.e., across campaigns and time periods). The results show that on average each campaign is both positively and negatively affected by the existence of other campaigns supporting relief efforts for the same natural disaster event. Specifically, the results in Table 4 indicate that an increase in the number of campaigns supporting relief efforts for a particular natural disaster event (NumCampaigns) leads to less donations received by each campaign with similar causes (DonationRcv) on average, consistent with Hypothesis 1.

The positive estimate for EarnRatio in Table 4 indicates that the relative success of the top earning campaigns on a particular day has positive externalities on other campaigns supporting relief efforts for the same event on the subsequent day. This result is consistent with Hypothesis 2. These results suggest that market expansion occurs only when some campaigns raising funds for relief efforts of a particular disaster event perform exceptionally well compared to other campaigns associated with the same event, consistent with [10] and [16]. A mere increase in the number of campaigns do not appear to expand the donors market. Instead, it has a negative effect on the amount of donations received by these campaigns.

Table 4. The effects of other campaigns

\begin{tabular}{lc}
\hline & $\log ($ DonationsRcv $)$ \\
\hline EarnRatio $(\mathrm{t}-1)$ & $22.193 * * *$ \\
& $(2.8603)$ \\
NumCampaigns $(\mathrm{t})$ & $-0.012 * * *$ \\
& $(0.0011)$ \\
\hline Observations & 86,678 \\
R-squared & 0.522 \\
\hline Note: ${ }^{* * *} \mathrm{p}<0.01, * * \mathrm{p}<0.05, * \mathrm{p}<0.1$. Robust standard \\
errors in parentheses. Coefficients for campaign fixed \\
effects are suppressed. Control variables are included \\
in the regression, but their estimates are suppressed. \\
\hline
\end{tabular}

Figure 3 shows that the positive effect of the relative success of the top earning campaigns is decreasing over time. However, this positive effect stays persistent for more than a week indicating that all campaigns supporting relief efforts for a particular natural disaster event continue to benefit from the relative success of the top earning campaigns.

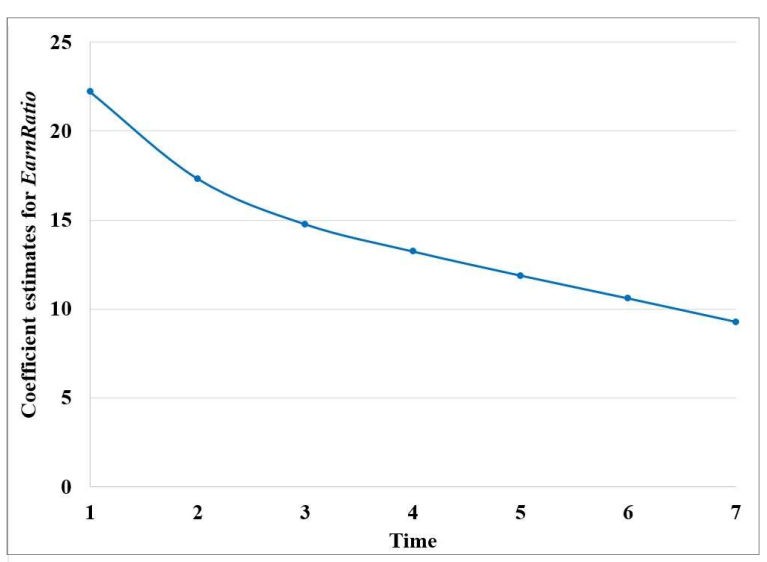

\section{Figure 3. Coefficient estimates for EarnRatio for different time lag}

Now the question is whether the existence of other campaigns with similar causes affect top performing campaigns versus low performing campaigns differently. Column 1 of Table 5 shows the estimates for the top $25 \%$ highest earning campaigns and column 2 of Table 5 shows the estimates for the bottom $75 \%$ lowest earning campaigns.

The results in Table 5 shows that negative and positive externalities are experienced by top earning campaigns as well as low earning campaigns. However, the negative externalities from an increased in the number of campaigns supporting relief efforts for the same event seems to be stronger for the top earning campaigns.

As expected, the relative success of top earning campaigns has positive externalities on those campaigns themselves on the subsequent day (column 1 of Table 5). However, more interestingly, positive externalities for other campaigns - i.e., campaigns with relatively weak performance so far - are observed. Specifically, the low performing campaigns also benefit from the relative success of top earning campaigns (column 2 of Table 5). 
Table 5. The effects of other campaigns, for top versus bottom earning campaigns

\begin{tabular}{lcc}
\hline & $\begin{array}{c}\text { Top 25\% } \\
\text { earners }\end{array}$ & $\begin{array}{c}\text { Bottom 75\% } \\
\text { earners }\end{array}$ \\
\hline & \multicolumn{2}{c}{$\log ($ DonationsRcv) } \\
\hline EarnRatio $(\mathrm{t}-1)$ & $37.916^{* * *}$ & $11.942 * * *$ \\
& $(5.4313)$ & $(3.5875)$ \\
NumCampaigns $(\mathrm{t})$ & $-0.013 * * *$ & $-0.009 * * *$ \\
& $(0.0014)$ & $(0.0011)$ \\
\hline Observations & 45,010 & 41,668 \\
R-squared & 0.539 & 0.423 \\
\hline Note: $* * * \mathrm{p}<0.01, * * \mathrm{p}<0.05, * \mathrm{p}<0.1$. Robust standard errors \\
in parentheses. Coefficients for campaign fixed effects are \\
suppressed. Control variables are included in the regression, but \\
their estimates are suppressed.
\end{tabular}

The regressions also include various control variables, whose parameter estimates are suppressed in Tables 3, 4, and 5. The arrivals of new disaster events do not significantly affect the donations received by top performing campaigns. However, these arrivals negatively affect the donations received by the non-top performing campaigns. This suggests that when a new natural disaster occurs, potential donors become less likely to support campaigns related to existing events that have not been performing well.

\section{Additional results}

One may also ask whether the existence of other campaigns affects the distribution of donations across campaigns supporting relief efforts for the same natural disaster event. More specifically, one may ask how the existence of other campaigns supporting similar causes affects the market share (will be called donation share, henceforth) of each campaign.

Table 6 shows the regression estimates for the empirical model in Equation 1 with a different dependent variable. In the modified specification, the share of donations (DonationShare) that a campaign has received thus far is used as the dependent variable. DonationShare is defined following the market share definition in [6], which is the portion of the total donations received by campaigns supporting a particular set of causes that is received by a particular campaign supporting one of those causes. By using donation share as a dependent variable in the model, we can examine whether the presence of other campaigns change how total donations for a particular event are distributed across campaigns supporting relief efforts for that event within the same platform.
Table 6. The effects of other campaigns on donation shares

\begin{tabular}{lcc}
\hline & $\begin{array}{c}\text { Top 25\% } \\
\text { earners }\end{array}$ & $\begin{array}{c}\text { Bottom 75\% } \\
\text { earners }\end{array}$ \\
\hline EarnRatio (t-1) & 0.012 & $-0.004^{* *}$ \\
& $(0.0105)$ & $(0.0018)$ \\
NumCampaigns $(\mathrm{t})$ & $-0.000^{* *}$ & $-0.000^{* *}$ \\
& $(0.0000)$ & $(0.0000)$ \\
\hline Observations & 44,672 & 40,455 \\
R-squared & 0.268 & 0.136 \\
\hline Note: $* * * \mathrm{p}<0.01, * *$ p $<0.05, * \mathrm{p}<0.1$. Robust standard errors \\
in parentheses. Coefficients for campaign fixed effects are \\
suppressed. Control variables are included in the regression, but \\
their estimates are suppressed.
\end{tabular}

The results in column 1 of Table 6 suggest that the presence of other campaigns supporting similar cause does not significantly alter the distribution of donations across top earning campaigns supporting relief efforts for a particular event. Furthermore, the distribution of donations across these top performing campaigns is also not affected by the relative success of these top performing campaigns.

In contrast, the results in column 2 of Table 6 indicate that the market shares of the low performing campaigns are negatively affected by the relative success of the top earning campaigns. It appears that while the low performing campaigns benefit from the relative performance of the top earning campaigns (see column 2 of Table 5), these low performing campaigns lose some of their market shares to the top performing campaigns.

\section{Robustness tests}

\subsection{Alternate measure of campaigns' performance}

Table 7 shows the regression estimates for another alternative specification of the empirical model in Equation 1. Social networks have been documented by existing studies to influence the success of noncharitable crowdfunding projects (e.g., [9], [13]). Donors who have contributed to campaigns on a particular platform can reach out to their social networks through various means of communication, including social media, advocating the platform as a viable channel for supporting charitable causes. This advocacy can increase the public awareness of the platform as a channel for charitable contributions.

In this modified model, MentionRatio is used to capture the relative popularity of the top endorsed 
campaigns in Facebook mentions and Twitter tweets on a particular day. MentionRatio is defined as the ratio of the total number of social media mentions received by the top endorsed campaigns supporting similar causes on a particular day over the total amount of social media mentions received by all campaigns supporting these causes on that day. ${ }^{2}$ The Facebook mentions and Twitter tweets received by the top endorsed campaigns can help advocate for the causes supported by those campaigns and the platform as a viable channel for supporting those causes.

The negative and positive externalities observed previously remains robust in this modified model. Similar to the results in Table 4 , the positive estimate for MentionRatio indicates that the relative success of the top endorsed campaigns in getting mentioned on social media has positive externalities on all campaigns supporting relief efforts for the same natural disaster event.

Table 7. The effects of other campaigns, using an alternate measure of campaigns' performance

\begin{tabular}{lc}
\hline & $\log ($ DonationsRcv $)$ \\
\hline MentionRatio $(\mathrm{t}-1)$ & $20.571^{* * *}$ \\
& $(2.6242)$ \\
NumCampaigns $(\mathrm{t})$ & $-0.011^{* * *}$ \\
& $(0.0010)$ \\
\hline Observations & 86,678 \\
$\mathrm{R}-\mathrm{squared}$ & 0.523 \\
\hline Note: $* * * \mathrm{p}<0.01, * * \mathrm{p}<0.05, * \mathrm{p}<0.1$. Robust \\
standard errors in parentheses. Coefficients for \\
campaign fixed effects are suppressed. Control \\
variables are included in the regression, but their \\
estimates are suppressed. \\
\hline
\end{tabular}

\subsection{Alternate specification on top and bottom performing campaigns}

The robustness of this study's main results in Table 4 is also checked by changing the specification for top earning campaigns. In this alternate specification, top earning campaigns are defined as the top $40 \%$ of campaigns with the highest donations received thus far. As such, EarnRatio40 is the ratio of the total donations received by the top $40 \%$ highest earning campaigns supporting the relief efforts of a specific natural disaster event on a particular day over the total donations received by all campaigns focusing on that event on the same day. Additionally, I use a subset of the original dataset containing only the top $40 \%$

\footnotetext{
2 Top endorsed campaigns are defined as campaigns that receive daily social media mentions that are in the top $25 \%$ of all
}

highest earning campaigns and the lowest $40 \%$ earning campaigns of the day in this regression. The middle $20 \%$ of campaigns are not included in this regression to exclude the effects on the $20 \%$ campaigns in the middle from the results.

The results in Table 8 shows that the direction and statistical significance of our variable of interests remain robust with this alternate specification of top earning campaign using the subset of dataset containing only the top and bottom $40 \%$ of campaigns based on the total donations they have received.

Table 8. The effects of other campaigns
using an alternate specification of top
earning campaigns and subset dataset

\begin{tabular}{lc}
\hline & $\log ($ DonationsRcv $)$ \\
\hline EarnRatio40 $(\mathrm{t}-1)$ & $49.219^{* * *}$ \\
& $(5.7025)$ \\
NumCampaigns $(\mathrm{t})$ & $-0.015^{* * *}$ \\
& $(0.0012)$ \\
\hline Observations & 71,235 \\
R-squared & 0.524 \\
\hline Note: *** $<<0.01, * * \mathrm{p}<0.05,{ }^{*} \mathrm{p}<0.1$. Robust \\
standard errors in parentheses. Coefficients for \\
campaign fixed effects are suppressed. Control \\
variables are included in the regression, but their \\
estimates are suppressed.
\end{tabular}

\section{Discussion and future directions}

This study finds both negative and positive externalities from the existence of other charitable campaigns supporting relief efforts for the same natural disaster event on the same platform. An increase in the number of campaigns supporting relief efforts for a particular event on the same crowdfunding platform leads to less donations received by those campaigns on the following day. This finding is consistent with the business stealing effects of additional campaigns.

However, when the top performing campaigns (in terms of donations or social media mentions) are performing exceptionally well, other campaigns supporting relief efforts for the same event on the same platform receive more donations on the following day. This study finds that the top performing campaigns are not the only ones benefiting from their good performance on the previous day. The low performing campaigns also benefit from abnormally good performance of the top campaigns. Our findings

campaigns supporting a set of similar causes (i.e., relief efforts in response to a specific natural disaster event). 
suggest that this positive externality of the abnormally good performing of the top campaigns appear to be fairly persistence over time.

This study also finds that the presence of other campaigns does not change how the donations are distributed across the top performing campaigns. However, the presence of other campaigns seems to reduce the donation share of the low performing campaigns. In other words, the low performing campaigns seem to lose the proportion of the total donations received by all campaigns supporting relief efforts for a particular natural disaster event even though these low performing campaigns receive more donations as a result of abnormally good performance of the top campaigns.

This study contributes to the literature on crowdfunding by documenting that the existence of multiple campaigns supporting similar causes on the same platform can generate externalities for other campaigns on that platform. An increase in the number of campaigns generates negative externality in the form of lower average donations. This negative effect suggests that a mere increase in the number of campaigns does not expand the donors' market.

The expansion of the donors market, in the form of more donations, appear to occur when the top campaigns supporting a particular cause perform exceptionally well. The positive effect of the exceptionally good performance of the top campaigns appears to benefit all campaigns, including the low performing ones.

\section{References}

[1] S. Adams, "Free Market Philanthropy: GoFundMe Is Changing The Way People Give To Causes Big And Small", Forbes.com, 2016 (available at https://www.forbes.com/sites/susanadams/2016/10/19/freemarket-philanthropy-gofundme-is-changing-the-waypeople-give-to-causes-big-and-small/; retrieved on May 18, 2018).

[2] G. Aldashev, and T. Verdier, "Goodwill Bazaar: NGO Competition and Giving to Development," Journal of Development Economics 91(1), 2010, pp. 48-63.

[3] M. Armstrong, "Competition in Two-sided Markets," The RAND Journal of Economics 37(3), 2006, pp. 668-691.

[4] P. Belleflamme, N. Omrani, and M. Peitz, "The Economics of Crowdfunding Platforms," Information Economics and Policy 33, 2015, pp. 11-28.

[5] H. Choi, and H. Varian, "Predicting the Present with Google Trends," Economic Record 88(s1), 2012, pp. 2-9.
[6] Cooper, L.G., and M. Nakanishi, Market-share Analysis: Evaluating Competitive Marketing Effectiveness, Kluwer Academic Publishers, Boston, 1988.

[7] P. Davis, "Measuring the Business Stealing, Cannibalization and Market Expansion Effects of Entry in the US Motion Picture Exhibition Market," The Journal of Industrial Economics 54(3), 2006, pp. 293-321.

[8] P. Gomber, R.J. Kauffman, C. Parker, and B.W. Weber, "On the Fintech Revolution: Interpreting the Forces of Innovation, Disruption, and Transformation in Financial Services," Journal of Management Information Systems, 35(1), 2018, pp. 220-265.

[9] M. Lin, N.R. Prabhala, and S. Viswanathan, "Judging Borrowers by the Company They Keep: Friendship Networks and Information Asymmetry in Online Peer-topeer Lending," Management Science, 59(1), 2013, pp. 1735 .

[10] M. Lin, R. Wu, and W. Zhou, "Two-Sided Pricing and Endogenous Network Effects," Working paper, 2016 (available https://papers.ssrn.com/sol3/papers.cfm?abstract_id $=24260$ $33)$.

[11] P. Ly, and G. Mason, "Competition between Microfinance NGOs: Evidence from Kiva." World Development 40(3), 2012, pp. 643-655.

[12] C. Mercer, "10 of the Best Crowdfunding Websites: Which Crowdfunding Site is the Best? How to Get Peer to Peer Funding," Techworld, 2017 (available at https://www.techworld.com/picture-gallery/startups/10-topcrowdfunding-websites-2016-3639335/; retrieved on March 19, 2018).

[13] E. Mollick, "The Dynamics of Crowdfunding: An Exploratory Study," Journal of Business Venturing 29(1), 2014, pp. 1-16.

[14] D. Ribar, and M. Wilhelm, "Altruistic and Joy-ofGiving Motivations in Charitable Behavior," Journal of Political Economy 110(2), 2002, pp. 425-457.

[15] J. C. Rochet, and J. Tirole, "Platform Competition in Two-sided Markets," Journal of the European Economic Association 1(4), 2003, pp. 990-1029.

[16] J. Wang, "Essays on Quality Screening in Two-Sided Markets," Doctoral dissertation, 2015, University of Southern California. 